\title{
EDITORIAL
}

\section{Geriatric Psychiatry: What's in a Name, and Five Concerns about DSM-5}

DOI:http://dx.doi.org/10.5770/cgj.17.117

Up until last year, the term "Geriatric Psychiatrist" was open to any psychiatrist in Canada who practiced with older adults. The new Royal College subspecialty is an important step in accrediting, training, and acknowledging the specialized work involved in geriatric psychiatry. No transition, however, can be entirely smooth. There is a dire need for expanded training positions and funding for these positions. Some senior members of our profession remain uncertain whether it is worth it to pursue this certification, which includes the need for an examination. It is possible that the subspecialty designation will be a requirement in certain jurisdictions, and there may be fee-code implications in the future, although uncertainty remains. The CAGP has offered a national review course and online study group for the last two years, and we have now tailored this for geriatricians, general psychiatrists, and other non-psychiatry stakeholders.

Another change for geriatric psychiatry, which has been perceived by the media as huge, was the introduction in May 2013 of the Diagnostic and Statistical Manual of Mental Disorders, 5th edition (DSM-5). ${ }^{(1)}$ I believe it is worthwhile to reflect on some of these changes further, as any change to diagnostic classification has important potential implications for clinical practice and our patients. The publication of the DSM-III in 1980 introduced standardized criteria for the diagnosis of mental disorders. This improved the reliability of psychiatric diagnoses over earlier classifications, which were guided by clinical impression in a more idiosyncratic fashion. While many biomarkers associated with a variety of mental disorders have been identified since the introduction of DSM-III, there are still no clinical laboratory tests or alternative "gold standards" for diagnosing mental disorder. ${ }^{(2)}$

Overall, the DSM-5 categorical diagnostic scheme is largely unchanged from DSM-IV, with more similarities than differences compared with the recent previous editions from DSM-III onward. Nonetheless, DSM-5 has eliminated the multi-axial scheme in which personality disorders, medical illness, psychosocial stressors, and a global functioning score were each listed on different "axes" from primary psychiatric disorders. Substance dependence and abuse have been simplified into a single substance use disorder. It was recognized that patients with delusional disorder do not necessarily have "non-bizarre" delusions, and that patients with anxiety disorders may not necessarily see their worries as "excessive or unreasonable". A few new disorders, like hoarding disorder and gambling disorder, have been introduced formally into the nomenclature, and this will likely be important for a small subgroup of older adults. There are other, more minor changes, which I will not summarize here, but these are my five biggest concerns or hesitations about the DSM-5 changes as they pertain to the practice of geriatric psychiatry:

1. A new diagnosis called "somatic symptom disorder" is meant to replace a variety of DSM-IV somatoform disorders. To meet criteria for this disorder, a patient must have one or more physical symptoms that are distressing or result in significant disruption of daily life. Additionally, they must have excessive thoughts, feelings, and behaviour about the symptoms or associated health concerns, generally for six months or more. The changes were made because of the rarely used category of the DSM-IV somatoform disorders, and to move away from a prior emphasis on difficult-to-characterize "medically unexplained symptoms"(3). Some clinicians, particularly those working in primary or tertiary care medical settings, would be hard-pressed to find patients with physical symptoms that they are not overly concerned about. Although there is reasonable reliability, and although rates are reasonably low when specific questions are applied to ascertain the psychological aspects of the symptoms (less than $10 \%$ prevalence in a recent medically ill cohort), ${ }^{(3)}$ it remains to be seen whether in clinical practice, this category over-identifies those with physical symptoms as having a mental disorder.

2. Dementia has been re-named "major neurocognitive disorder", which introduces a supposedly more culturally sensitive term, but will likely confuse many. The body of the text of DSM-5 indicates that the term "dementia" may still be used, but I am uncertain as to who will read the entire body of the text of a book that is almost 1000 pages long. My patients and their family members have enough trouble understanding the differences between dementia and Alzheimer's disease. What will "neurocognitive disorder" mean to them?

3. A new diagnosis which was not listed in the main body of DSM-IV, called "mild neurocognitive disorder", has been introduced. Essentially this is fairly similar to the concept 
of mild cognitive impairment (or MCI) which is very familiar to most geriatricians and geriatric psychiatrists. The text of the DSM-5 indicates that performance 1 to 2 standard deviations below the mean in one cognitive domain (as established by several possible neurocognitive tests), in addition to subjective cognitive concerns or concerns of the informant or clinician about the patient's cognition, would qualify for this diagnosis. Research has shown that even subtle changes to the exact MCI definition has a dramatic impact on prevalence. ${ }^{(4)}$ In clinical settings, neuropsychological testing is not widely available. Although the DSM-5 indicates that "another quantified clinical assessment" can substitute when neuropsychological testing is not feasible, the details and cut-offs are not specified. It is clear that dementia is underdiagnosed, but pathologizing normative cognitive changes, which are present in the $16 \%$ of older persons that by definition score 1 standard deviation below the population mean, is unlikely to be helpful. Patients with other versions of $\mathrm{MCI}$ diagnoses so far have not been seen to respond to conventional pharmacotherapy for dementia the way patients with mild-to-moderate Alzheimer's disease do. ${ }^{(5,6)}$

4. The DSM-IV prevented the diagnosis a major depressive episode (MDE) within two months of the death of one spouse or a loved one unless serious functional impairment or unless specific complicated symptoms (e.g., psychomotor slowing, worthlessness, psychosis, and suicidal thoughts) were present. The authors of the DSM-5 have suggested that an MDE could be diagnosed at any time after a loss as long as criteria are met, essentially removing the "bereavement exclusion". Wakefield et al.(7) are among the authors who have demonstrated differences between bereavement complicated by major depression and bereavement uncomplicated by major depression. The authors demonstrated that there were three-fold more false positives (or lack of external validators) when the bereavement exclusion was changed from one year (in the DSM-III-R) to two months with the DSM-IV, and when one or more complicating symptoms (instead of two or more in the DSM-IIIR) could yield a major depression diagnosis; the false-positive rate rose from $6.2 \%$ to $28.4 \% .{ }^{(7)}$ It is possible that without the time exclusion, DSM-5 will be associated with more false positives, but this has not yet been studied. On the other hand, another recent large-scale study showed that the presence or absence of the bereavement exclusion did not predict antidepressant treatment response, ${ }^{(8)}$ so the relevance to treatment remains questionable. Furthermore, both of the bereavement studies that I have mentioned focused on younger adults, so it is unclear whether the situation is the same in the elderly.

5. The reliability of established experts in the field trials of DSM-5 was suboptimal, particularly for major depression, but also for minor neurocognitive disorder. (9) I would suspect that the inter-rater agreement of clinicians, who do not always use the criteria verbatim, would be even less. Although this was probably also a problem with prior editions of the DSM, there have been no direct comparisons.

It is easy for me to be an armchair critic of this process, and I can certainly say that I personally have no better solutions. Many parts of Canada do not and cannot approach the Mental Health Commission of Canada's benchmarks for staffing in geriatric psychiatry ${ }^{(10)}$ and an increase in false positives with the lower thresholds in DSM-5 could worsen this situation. On the flip-side, mental disorders in late life are woefully under-diagnosed, and if DSM had instead taken a more restrictive approach, that would have been perpetuated.

Hopefully we will see more studies about the reliability and external validity of the criteria published in the next several years which may provide reassurance and, ideally, there will be direct comparisons between new proposals with the current and recent editions. It will also be interesting to see whether, in practice, the newly introduced dimensional measures in the DSM-5, which are freely available online, ${ }^{(11)}$ turn out to be reliable, valid, helpful, practical, and sensitive to change, particularly in the older population.

\section{ACKNOWLEDGEMENTS}

The author acknowledges the valuable feedback of Dr. Benoit Mulsant, Dr. Corinne Fischer, Dr. Zahinoor Ismail, and Dr. Cindy Grief in the preparation of this editorial.

Mark J. Rapoport, MD, FRCPC Associate Professor, Department of Psychiatry University of Toronto, Sunnybrook Health Sciences Centre

\section{REFERENCES}

1. American Psychiatric Association. Diagnostic and statistical manual of mental disorders, 5th ed. Arlington, VA: American Psychiatric Association; 2013.

2. Paris J. The Intelligent clinician's guide to the DSM-5. New York: Oxford University Press; 2013.

3. Dimsdale JE, Creed F, Escobar J, et al. Somatic symptom disorder: an important change in DSM. J Psychosom Res. 2013;75(3):223-28.

4. Ganguli M, Chang CC, Snitz BE, et al. Prevalence of mild cognitive impairment by multiple classifications: the MonongahelaYoughiogheny Healthy Aging Team (MYHAT) project. Am J Geriatr Psychiatry. 2010;18(8):674-83.

5. Doody RS, Ferris SH, Salloway S, et al. Donepezil treatment of patients with MCI: a 48-week randomized, placebo-controlled trial. Neurology. 2009;72(18):1555-61. 
6. Tricco AC, Soobiah C, Berliner S, et al. Efficacy and safety of cognitive enhancers for patients with mild cognitive impairment: a systematic review and meta-analysis. CMAJ. 2013;185(16):1393-401.

7. Wakefield JC, Schmitz MF, Baer JC. Did narrowing the major depression bereavement exclusion from DSM-III-R to DSMIV increase validity?: evidence from the National Comorbidity Survey. J Nerv Ment Dis. 2011;199(2):66-73.

8. Corruble E, Falissard B, Gorwood P. Is DSM-IV bereavement exclusion for major depression relevant to treatment response? A case-control, prospective study. J Clin Psychiatry. 2011;72(7):898-902.

9. Regier DA, Narrow WE, Clarke DE, et al. DSM-5 field trials in the United States and Canada, Part II: test-retest reli- ability of selected categorical diagnoses. Am J Psychiatry. 2013;170(1):59-70.

10. Mental Health Commission of Canada. Guidelines for comprehensive mental health services for older adults in Canada. Ottawa: Mental Health Commission of Canada; 2013. Available from: http://www.mentalhealthcommission.ca/English/ discussion/4814/guidelines-comprehensive-mental-healthservices-older-adults-canada

11. American Psychiatric Association. Online assessment measures. Arlington, VA: American Psychiatric Association; [n. d.]. Available from: http://www.psychiatry.org/practice/dsm/dsm5/ online-assessment-measures 